\title{
Visual Prey Capture in Larval Zebrafish Is Controlled by Identified Reticulospinal Neurons Downstream of the Tectum
}

\author{
Ethan Gahtan, ${ }^{1,2,3}$ Paul Tanger, ${ }^{1}$ and Herwig Baier ${ }^{3}$ \\ ${ }^{1}$ Department of Psychology, University of Massachusetts, Amherst, Amherst, Massachusetts 01003, 2Department of Psychology, Humboldt State University, \\ Arcata, California 95521, and ${ }^{3}$ Department of Physiology, Program in Neuroscience, University of California, San Francisco, San Francisco, California \\ 94158-2722
}

\begin{abstract}
Many vertebrates are efficient hunters and recognize their prey by innate neural mechanisms. During prey capture, the internal representation of the prey's location must be constantly updated and made available to premotor neurons that convey the information to spinal motor circuits. We studied the neural substrate of this specialized visuomotor system using high-speed video recordings of larval zebrafish and laser ablations of candidate brain structures. Seven-day-old zebrafish oriented toward, chased, and consumed paramecia with high accuracy. Lesions of the retinotectal neuropil primarily abolished orienting movements toward the prey. Wild-type fish tested in darkness, as well as blind mutants, were impaired similarly to tectum-ablated animals, suggesting that prey capture is mainly visually mediated. To trace the pathway further, we examined the role of two pairs of identified reticulospinal neurons, MeLc and MeLr, located in the nucleus of the medial longitudinal fasciculus of the tegmentum. These two neurons extend dendrites into the ipsilateral tectum and project axons into the spinal cord. Ablating MeLc and MeLr bilaterally impaired prey capture but spared several other behaviors. Ablating different sets of reticulospinal neurons did not impair prey capture, suggesting a selective function of MeLr and MeLc in this behavior. Ablating MeLc and MeLr neurons unilaterally in conjunction with the contralateral tectum also mostly abolished prey capture, but ablating them together with the ipsilateral tectum had a much smaller effect. These results suggest that MeLc and MeLr function in series with the tectum, as part of a circuit that coordinates prey capture movements.
\end{abstract}

Key words: prey capture; tectum; reticulospinal; laser ablation; zebrafish; visuomotor

\section{Introduction}

Visuomotor behaviors depend on multisynaptic pathways that link the photoreceptors in the retina to muscle activity in the periphery. To elicit a particular behavior, stimulus features have to be recombined and channeled into separate neural pathways. For example, recognition and approach of prey are elicited by a distinct combination of stimulus cues, which have to be extracted rapidly and faithfully from a complex visual scene. The neuronal activity pattern signifying prey is conveyed from visual circuits to premotor circuits, which in turn send commands to motor neurons in the spinal cord. The ensemble of premotor neurons encoding identity and location of an external object and triggering an innate response to that object has been referred to in the ethological literature as a "command releasing system" (Ewert, 1987). Such a system may be exclusively dedicated to a particular behavior, although it is debated whether this is a general feature

Received Aug. 24, 2004; revised Aug. 18, 2005; accepted Aug. 22, 2005.

This work was funded by the National Institutes of Health, the Packard and Klingenstein Foundations (H.B.), and a National Research Service Award Postdoctoral Fellowship (E.G.). We thank members of our laboratory for discussions and Marta Gaglia for initial work on the prey capture assay.

Correspondence should be addressed to Ethan Gahtan, Department of Psychology, Humboldt State University, Arcata, CA 95521. E-mail: eg51@humboldt.edu.

D01:10.1523/JNEUROSCI.2678-05.2005

Copyright $\odot 2005$ Society for Neuroscience $\quad$ 0270-6474/05/259294-10\$15.00/0 of CNS organization (Metzner and Juranek, 1997; Edwards et al., 1999; Kupfermann and Weiss, 2001; Sewards and Sewards, 2002).

The larval zebrafish brain is well suited for an investigation of visuomotor coding (Gahtan and Baier, 2004). Zebrafish display several stereotyped responses to visual stimuli, and the neural substrate underlying these behaviors is composed of a small number of neuronal elements. Retinal ganglion cells (RGCs) project to 10 distinct areas in the brain (Burrill and Easter, 1994), consistent with functional segregation of behavioral pathways. Laser ablation of the optic tectum, the largest retinorecipient structure, leaves optomotor responses (OMRs) and optokinetic responses (OKRs) unaffected, two visuomotor reflexes that compensate for self-motion (Roeser and Baier, 2003). This result suggests that the individual visual pathways in these larval fish are already dedicated to processing behavior-specific information.

In zebrafish, motor commands generated in the brain are conveyed to the spinal cord through a small number of reticulospinal (RS) neurons. There are $\sim 150$ RS neurons, the cell bodies of which are arranged in a bilaterally symmetric array in midbrain and hindbrain. Few attempts have been made to link identifiable premotor systems to certain behaviors. Best known are the Mauthner neuron (or M cell) and its segmental homologs MiD2 and MiD3, which play a critical role in fast-start escapes (Zottoli et al., 1987; Faber et al., 1989; Eaton et al., 1991; Svoboda and Fetcho, 
1996; O’Malley et al., 1996; Liu and Fetcho, 1999; Eaton et al., 2001; Zottoli et al., 1987). However, even the apparently simple startle circuit is complex: stimuli eliciting escapes activate many other RS neurons in addition to these three pairs (Gahtan et al., 2002), and even triple ablations do not eliminate startle responses altogether (Liu and Fetcho, 1999).

Here, we investigated the neural substrate of capture of prey, a complex visually evoked behavior characterized by a unique pattern of axial muscle movements (Budick and O'Malley, 2000; Borla et al., 2002). We discovered that the tectum, in conjunction with two identified pairs of RS neurons, MeLr and MeLc, are required for normal prey capture performance. Our studies have allowed us to identify essential neuronal elements of a dedicated visuomotor pathway in the vertebrate brain.

\section{Materials and Methods}

Animals. Zebrafish of the Tübingen Long and Long-Finned Gold strains were kept and bred according to standard procedures. Larvae were raised in $9 \mathrm{~cm}$ Petri dishes at a density of $<40$ per dish. A Brn3c:mGFP transgenic line was used for laser ablations and behavioral experiments. In this line, membrane-bound green fluorescent protein (GFP) is expressed in approximately one-half of all RGCs, including those projecting to the stratum fibrosum et griseum superficiale of the tectum, and in mechanosensory hair cells in the skin and the inner ear (Xiao et al., 2005). Furthermore, a Shh:GFP transgenic line (Neumann and NuessleinVolhard, 2000) was used to visualize RGC axons simultaneously with backfilled RS neurons. Shh:GFP labels all RGC types, in addition to subpopulations of neurons in the ventral thalamus (Roeser and Baier, 2003). Depending on the experiment, GFP was imaged either with a confocal laser-scanning microscope (MRC-1024; Bio-Rad, Hercules, CA), a compound fluorescence microscope (Axioskop II; Zeiss, Oberkochen, Germany), or an epifluorescence-equipped dissecting microscopem (MZ FLIII; Leica, Nussloch, Germany).

Retrograde labeling of RS neurons. Zebrafish larvae, aged 4-5 d postfertilization $(\mathrm{dpf})$, were transferred into embryo medium containing $0.03 \%$ 3-aminobenzoic acid ethyl esther methane sulfonate (MESAB; Sigma, St. Louis, MO) to anesthetize them before tracer injections. Anesthetized larvae were placed into an agar-coated Petri dish, and a glass injection pipette containing the tracer solution was inserted, under a dissecting light microscope, through the lateral muscle and into the ventral spinal cord. The injection was at the level of the 20th myotome, approximately three-quarters down the length of the spinal cord. Care was taken to avoid penetrating intersegmental blood vessels. For ablations, a $0.75 \%$ solution (w/v) of Alexa Fluor dextran [molecular weight (MW), 10,000; Invitrogen, San Diego, CA] was injected, and, for confocal anatomy studies, a $0.5 \%$ solution of Texas Red dextran (MW, 10,000; Invitrogen) was used. Tracers were pressure ejected from the pipette tip using a PicoPump (World Precision Instruments, Sarasota, FL). These tracers enter RS neurons through severed axonal processes at the spinal injection site (Gahtan and O'Malley, 2003). At least $12 \mathrm{~h}$ were allowed for retrograde filling of hindbrain cell bodies and dendrites. Multiple descending neurons, on both sides of the hindbrain and midbrain, were labeled in most injected larvae. The present study focused on two pairs of individually identifiable descending neurons in the nucleus of the medial longitudinal fasciculus (nMLF), because their anatomy suggested that they may receive tectal input. As a control for bilateral ablation of the MeLr and MeLc neurons, an equal number of RS neurons from outside of the nMLF were ablated. These control ablations targeted any four neurons among a subset of 14 individually identifiable neurons within rhombomeres 4-6, including the Mauthner neuron and its two segmental homologs. Because dye injections did not always label these cells of interest, injected fish were first screened for suitable labeling patterns.

Laser ablations. GFP-expressing larvae were sorted at 2 or $3 \mathrm{dpf}$. At 6 or $7 \mathrm{dpf}$, larvae were first anesthetized in a $0.03 \%$ MESAB solution and then embedded in $1.2 \%$ agar, dorsal side down, against a glass coverslip. The coverslip was then inverted onto a depression microscope slide so that the brain could be visualized through the dorsal surface of the head. Two methods of laser ablation were used, which gave similar results. Tectum ablations, and RS ablations done in conjunction with tectum ablation, were performed using a MicroPoint (Photonic Instruments, Arlington Heights, IL) laser system (equipped with a VSL-337ND-S nitrogen laser; Laser Science, Franklin, MA) on a Zeiss Axioskop II compound microscope. The primary UV laser pumped a $440 \mathrm{~nm}$ coumarin laser, which was aimed via a $20 \times$ (for tectum ablations) or $40 \times$ (for RS ablations) microscope objective (Plan-Neofluar 20×, numerical aperture, $0.5 ; 40 \times$, numerical aperture, 0.8 ; Zeiss). These ablations were performed under simultaneous visual control through GFP optics. Laser power was attenuated by at least $50 \%$ with neutral gray filters to minimize incidental tissue damage. The laser was used at a pulse rate of $2-10 \mathrm{~Hz}$. During the ablation procedure, the fish were repositioned to achieve the clearest view of targeted neurons or axons. Under these conditions, ablation of one tectal lobe took between 3 and $10 \mathrm{~min}$, and ablation of nMLF neurons took $\sim 1 \mathrm{~min}$ (see Results). Tectum and nMLF ablations were done either bilaterally or unilaterally, as well as alone or in combination. In experiments comparing the effects of nMLF ablation and ablation of non-nMLF RS neurons, ablations were performed using a confocal laser microscope (Leica TCS SL, with a $>30 \mathrm{~mW}$ laser intensity at the focal plane, using the $488 \mathrm{~nm}$ argon laser line; the confocal system was used in conjunction with a Leica DM LFS compound microscope equipped with a $40 \times$ water-immersion objective lens). In these ablations, a $488 \mathrm{~nm}$ laser was used in scanning mode and set at low intensity to identify and target individual RS neurons. The beam was then parked in a single position at the center of the targeted neuron and increased to maximum intensity. Under these conditions, it took $\sim 90$ s to ablate a single RS neuron.

Prey capture assay. Live paramecia cultures and protozoa food pellets were obtained from Carolina Biological Supply (Burlington, NC). Cultures were grown in $500 \mathrm{ml}$ plastic flasks, at $28.5^{\circ} \mathrm{C}$, to a density of $\sim 100$ paramecia per milliliter, and new cultures were started every 2-4 weeks.

The prey capture assay was run immediately after measurement of spontaneous activity (see below), and the groups of ablated and control larvae remained in the same $60 \mathrm{~mm}$ dishes for both procedures. Between 0.5 and $2.0 \mathrm{ml}$ of paramecia culture was added to the larvae's dishes to achieve a concentration of $\sim 50$ paramecia per fish. A similar volume was added to dishes with embryo medium, but no fish, to determine the effect of embryo medium on paramecia viability over the assay period. Each dish was placed on a glass plate and illuminated from below with a fiber optic cable, and video images of the dish were recorded with a high-speed digital camera positioned above the dish. Either a Motionscope PCI from Redlake Imaging (San Diego, CA) or a PL-A741 from PixeLINK (Ottawa, Ontario, Canada) was used. Two hundred video frames, captured at 60 $\mathrm{Hz}$, were recorded for each dish at each time point, and dishes were recorded successively, maintaining their serial order throughout the experiment.

An off-line imaging-based counting method was used to determine the number of paramecia in each dish immediately after they were added (time 0 ) and again every hour for $5 \mathrm{~h}$. Paramecia in healthy cultures remain in constant motion, allowing individual paramecia to be counted by tracking their path across the 200 video frames (encompassing $3.33 \mathrm{~s}$ ). The video frames were first projected using the $\mathrm{SD} z$-projection method in ImageJ, a java-based image-processing package developed and made freely available by the National Institutes of Health (for details on the projection method, see http://rsb.info.nih.gov/ij/). This procedure highlights changes in pixel values from one frame to the next and subtracts stable background pixels. This method allows the movement of paramecia and fish to be easily distinguished from each other and from background noise (see Fig. 1 A). Paramecia were marked and counted manually from the projected image, which was saved, whereas the larger movie file was deleted.

Results were expressed as the average number of paramecia per fish (calibrated to be $\sim 50$ ) relative to the same number at the starting time point. A decline in the percentage of counted paramecia over time could be caused by predation by the larvae as well as "natural" paramecia death (natural being defined here as "not by predation"). We therefore always ran a control plate, containing paramecia but not fish, alongside each experiment. The number of paramecia counted in experimental wells was adjusted at each time point to correct for the measured natural 
decline. Spontaneous paramecia death was very rarely $>5 \%$ and was never $>25 \%$. In some experiments, a "dark" condition was run to determine whether prey capture performance was vision dependent. In this case, dishes were placed in an opaque black plastic container and removed each hour for the brief ( $\sim 30 \mathrm{~s})$ imaging sessions.

Prey capture performance in ablated and control groups was analyzed using a repeated-measures ANOVA. The number of paramecia was counted six times for each plate, at time 0 , and once per hour for $5 \mathrm{~h}$, and therefore sampling time was analyzed as a within-subjects repeated measure. There was a significant main effect of group $\left(F_{(7,48)}=11.35 ; p<\right.$ $0.01)$, and a significant time by group interaction $\left(F_{(35,240)}=4.20 ; p<\right.$ $0.01)$, indicating that the groups differed in the rate of decline in paramecia. One-tailed Bonferroni's $t$ tests were used for subsequent pairwise comparisons of treatment groups at individual sampling points.

Analysis of prey encounter behaviors. To investigate how tectum and MeLr/MeLc ablation might disrupt prey capture performance, highspeed video recordings of individual prey encounter episodes were analyzed in separate groups of bilateral tectum ablated, bilateral MeLr/MeLc ablated, and injected control larvae (all were Brn3c:mGFP transgenics). Two larvae from each treatment condition were placed in $35 \mathrm{~mm}$ clear Petri dishes containing $\sim 3 \mathrm{ml}$ of embryo medium. The video recording method and apparatus were the same as that described for paramecia counting experiments, except that the camera was set to record for the period preceding a manual trigger. Between 15 and 30 paramecia were added to a dish, after which the dish was observed continuously for $1 \mathrm{~h}$ using a video preview monitor attached to the camera (the camera itself remained in record mode). Ten prey encounter episodes were recorded for later analysis. A prey encounter episode was defined as any time at which a paramecia moved within a semicircular area extending $5 \mathrm{~mm}$ around the head of the larva (with the straight edge of the semicircle running between the two eyes), followed by movement of the fish. These criteria were based on our previous observations of prey capture episodes and visual orienting responses in control larvae and probably represent a conservative estimation of the fish's visual abilities. Analysis of prey encounters was according to Borla et al. (2002). The larvae's head yaw (the angular displacement of the head during successive side-to-side bends of a swim bout) and the fish-to-paramecium distances were measured using automated image analysis procedures in ImageJ.

Locomotor activity assay. Because prey capture requires swimming, spontaneous activity was measured to determine whether laser ablations of the tectum or the nMLF disrupted locomotor performance. Spontaneous activity was tested in groups of between three and six individual larvae, aged 7-8 dpf. Each group was maintained in a $60 \mathrm{~mm}$ Petri dish containing $\sim 6 \mathrm{ml}$ of embryo medium. Each larva within a group received the same treatment: bilateral tectum ablation, bilateral nMLF ablation, or control. Because ablations required fluorescent prelabeling of the targeted structure (tectum, nMLF, or both), larvae in control groups were also imaged to ensure that they showed a fluorescent signal in the corresponding structure. In the case of tectum ablations, this meant that control larvae were all Brn3c:mGFP transgenics. In the case of nMLF ablations, control larvae had received spinal injection of fluorescent tracer and were confirmed to have labeled neurons in the nMLF. All control larvae were siblings of ablated larvae and received identical treatment with the exception of the ablation procedure.

Dishes of ablated and control larvae were placed on a glass plate positioned above a digital camcorder (TRV-9; Sony, Tokyo, Japan). Lightdiffusing plastic above the dishes produced a uniform light background in recorded images. Images were captured directly to the computer at a rate of $0.5 \mathrm{~Hz}$ for $20 \mathrm{~min}$, creating a 600 frame movie file. Images were recorded using Adobe Premiere software (Adobe Systems, San Jose, CA) and analyzed using ImageJ. For the analysis, pixel values from pairs of successive movie frames were subtracted such that only changes caused by fish movements or random noise remained. After this background subtraction, the software could be configured to locate and count all of the fish in a frame, based on their characteristic size and contrast (specified in pixel area and pixel intensity, respectively). For each pair of frames, only fish that moved were counted, because stationary pixels were subtracted out. Individual fish within a group could not be distinguished in this analysis; therefore, activity was measured for the group as a whole, specifically, results were expressed as the percentage of larvae within the group that moved from one frame to the next, averaged across the 20 min (600 frame) observation period.

OKR assay. The OKR was tested in larvae with unilateral nMLF lesions, using previously published procedures (Roeser and Baier, 2003; Orger et al., 2004). Briefly, a computer-generated image of a rotating black and white grating was projected onto the inner walls of a white drum (height, $50 \mathrm{~mm}$; inner diameter, $56 \mathrm{~mm}$ ). Larvae were mounted in methyl cellulose in a $30 \mathrm{~mm}$ Petri dish, dorsal up and facing out toward the moving grating. Stimulus velocity for the sine wave grating was $20^{\circ}$ per second. Stimuli were presented to one eye only by restricting the stimulus to $180^{\circ}$ of the visual field, and each eye was tested on both temporal-to-nasal and nasal-to-temporal stimulus directions. Eye positions of individual fish were recorded onto a computer at four frames per second. Eye movements of the stimulated eye only were plotted as angle (relative to the axis of the fish) over time, and the saccade rate was measured manually in representative parts of the OKR trace. Paired-samples $t$ tests were used to compare saccade rates between ablated and intact sides within fish. Fish tested in the OKR assay were not used in other behavioral tests.

\section{Results}

\section{Prey capture is a coordinated series of actions controlled by visual feedback}

We observed in high-speed video recordings that zebrafish larvae often detected a paramecium from a distance and then oriented toward it, bringing the prey object in front of their mouth. The fish then approached and, if necessary, pursued, and swallowed the paramecium (Fig. 1) (Budick and O’Malley, 2000). Paramecia of the variety used in this study swam steadily at a rate of $\sim 3$ $\mathrm{mm} / \mathrm{s}$. Zebrafish larvae occasionally chased a paramecium over distances of several body lengths, making a variable number of turns as needed, before they finally ingested it (Fig. $1 B$; see supplemental movie of this particular episode, available at www.jneurosci.org as supplemental material). These observations indicate that the appetitive phases of this behavior are under continual control of sensory, presumably visual, feedback.

\section{A novel behavioral assay records prey capture performance by zebrafish larvae}

We devised a prey capture assay that allowed us to record the rate of predation by zebrafish larvae under different experimental conditions, as described in Materials and Methods. Briefly, we added $\sim 50$ paramecia per fish to dishes containing three to six fish larvae and counted their number every hour for $5 \mathrm{~h}$ (Figs. 1, 2 ). The decline in paramecia number, corrected for their natural disappearance when no fish was present, was directly related to the efficiency by which zebrafish consumed them. We found that paramecia were eaten at a steadily declining rate, after a negative exponential with a half time of $\sim 2 \mathrm{~h}$. The gradual drop in the rate of prey catching is probably best explained by the steady decrease in the density of paramecia. Satiety may also be a factor at later sampling points. The new assay provided a rapid, robust, and quantitative readout of prey capture by larval zebrafish.

\section{Prey capture primarily depends on an intact optic tectum and is not lateralized}

To begin to explore the neural substrates of prey capture behavior, we first tested whether the optic tectum was involved, as was reported for other vertebrates in various food-seeking behaviors. For this experiment, we laser ablated the retinotectal neuropil in transgenic zebrafish whose RGC axons were labeled with GFP (Fig. 2A). This method is able to remove retinotectal visual input, while leaving other retinofugal projections (e. g., to the pretectum, thalamus, and hypothalamus) intact (Roeser and Baier, 2003) (the effectiveness of the ablations was demonstrated by 


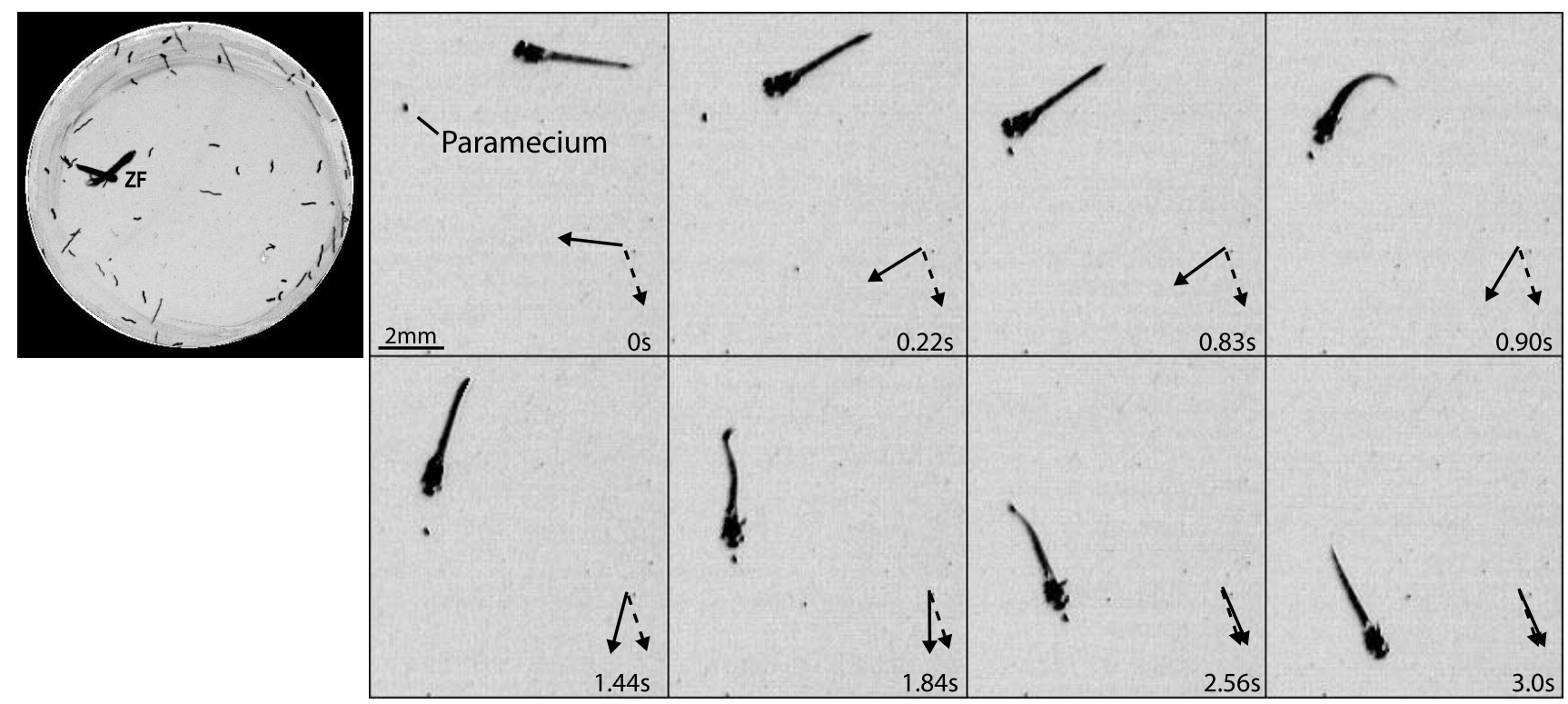

Figure 1. Measuring prey capture in larval zebrafish. $A$, Projection of a time-lapse image series showing a zebrafish and paramecia during the prey capture assay. Dark streaks within the circular Petri dish are the travel paths of individual paramecia during the 3.3 s of imaging ( 200 frames at 60 frames per second). The movement path of a zebrafish larva ( $(\mathrm{F})$ can be distinguished clearly from the paramecia. $\boldsymbol{B}$, Time series showing a zebrafish larva capturing a paramecium. The sequence spans $3 \mathrm{~s}$, and the eight frames shown were chosen to highlight key features of the prey capture behavior, including orienting turns, approach swims $(0-0.83 \mathrm{~s})$, pursuit swims $(1.44-2.56 \mathrm{~s})$, and the final capture swim $(2.56-3.0 \mathrm{~s})$. The dashed arrow shows the heading of the paramecium, measured as a line drawn from its start point to its point at time of capture. The paramecium swims in a straight line during this $3 \mathrm{~s}$ period. The solid arrow shows the heading of the zebrafish larva, measured as the orientation of the long axis of the head. As the larva pursues the paramecia, its orientation aligns with the heading of the paramecia, and the two arrows gradually converge.

postablation dye injections into the eye, which labeled pretectal retinofugal projections but not those in the tectum). GFPtransgenic sibling larvae served as controls. We found that fish with bilateral tectum lesions consumed substantially fewer paramecia than unablated animals (Fig. 2 B, unablated controls, labeled GFP+Light). Post hoc comparisons showed that the bilateral ablation group had less overall decline in paramecia than the unablated control group (mean difference, 27.3; $p<0.01$ ), and this difference was significant at all sampling points except the start point.

When bilaterally tectum-ablated fish were present, paramecia numbers dropped at a rate of $\sim 12 \%$ per hour, compared with $40 \%$ per hour in controls. The paramecia capture rate by unilaterally tectum-ablated fish was intermediate between the control and bilaterally ablated groups at each sampling point ( $\sim 25 \%$ per hour) (Fig. 2B). This effect was symmetric with regard to the left or the right tectum (Fig. $2 B$, data shown for the $5 \mathrm{~h}$ sampling point only) (the overall mean difference in paramecia consumption between the left and right tectum ablation groups was 5.9\%). The fact that ablation of either the left or the right tectum resulted in a similar impairment suggests that prey capture is not lateralized in larval zebrafish. The natural decline of paramecia in the absence of any fish was very rarely $>5 \%$ per hour, which is substantially less than when bilateral tectum-ablated fish were present (12\% per hour). This suggests that tectum-ablated animals were still able to consume prey. In summary, these lesion experiments demonstrated that paramecia capture depends, to a large extent, albeit not exclusively, on an intact optic tectum.

\section{Prey capture is primarily driven by vision}

From our high-speed video recordings, it appeared that zebrafish larvae use their visual sense to detect and to pursue paramecia. The results of tectum ablations are also consistent with a prominent role for vision; however, they do not exclude a major con- tribution by other sensory modalities, because the tectum is known to receive input from several other sensory systems. We therefore tested the role of vision in our prey capture assay using two independent methods. First, we measured paramecia consumption by normal zebrafish larvae held in constant darkness (Fig. $2 B$, the group labeled GFP+ Dark). Although the initial decline in paramecia was $40 \%$ per hour for the fish in the light, it was only $\sim 10 \%$ for fish kept in darkness. The average for the dark group was significantly lower at each sample point (Fig. 2 B). Interestingly, the bilaterally tectum-ablated group did not differ significantly from the dark-kept group (Fig. $2 B$ ), indicating that most if not all of the vision-driven prey capture depends on the tectum. Second, we tested prey capture performance in a completely blind mutant line, lakritz, which lacks RGCs but is otherwise normal and viable (Kay et al., 2001). lakritz mutants were significantly impaired relative to wild-type siblings tested in normal ambient light and were very similar to wild-type siblings kept in darkness [overall mean difference from sibling control, 33\% $(p<0.01)$ and $37 \%(p<0.01)$ for dark-kept siblings and mutants, respectively] (Fig. $2 C$ ). These results confirm that prey capture depends, to a large degree, on vision.

\section{MeLr and MeLc are selectively involved in visual prey capture} We next asked whether we could delineate the prey-capture pathway further, beyond the tectum and into premotor areas of the brain. Tectal cells do not extend projections into the spinal cord, but they do form monosynaptic connections with RS cells that do so. Among them are the M cell, as well as neurons in the nMLF, located ventrally to the tectum. The $\mathrm{M}$ cell has been shown by others to be dispensable for prey capture (Borla et al., 2002). The nMLF, in contrast, had not been tested previously. We decided to focus on those neurons in the nMLF that are tectorecipient and that, at the same time, project axons to the spinal cord.

Neurons in the nMLF were backfilled from the spinal cord 
A
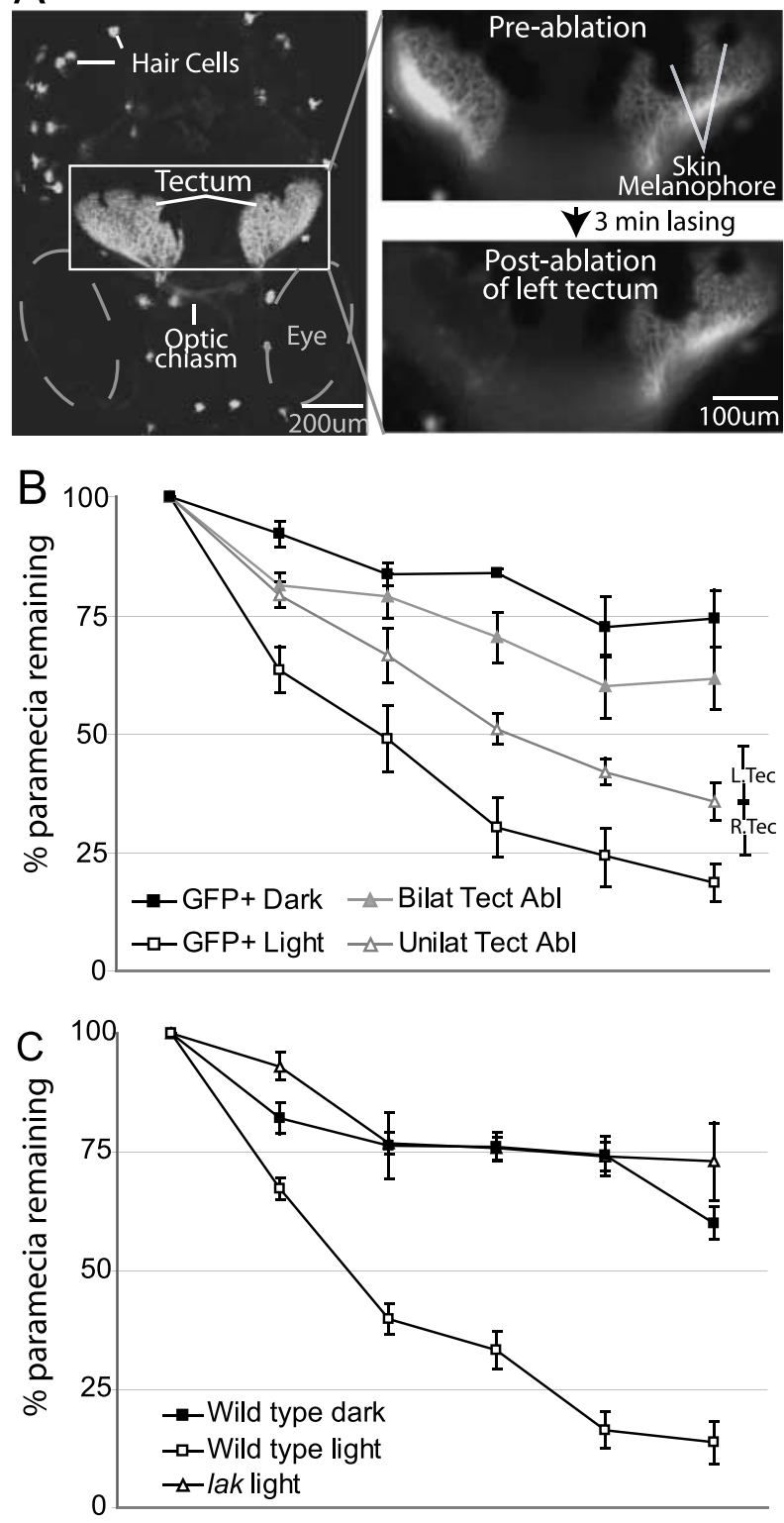

Figure 2. Prey capture requires the tectum and is vision dependent. $\boldsymbol{A}$, Laser ablation of the tectum in GFP-expressing larvae. The left panel is a projection of a confocal image stack showing GFP expression in RGCs terminating at the tectum in Brn3c:mGFP transgenic larvae (20 images taken at $2 \mu \mathrm{m}$ intervals). The optic chiasm and GFP-expressing hair cells are also visible. The right panels show an enlargement of the boxed section containing the tectum on both sides. These are single images acquired under epifluorescence, showing the same section before (top) and after (bottom) laser ablation of the left tectum. After 3 min of visually guided lasing, GFP expression is no longer visible in the left tectum but is unchanged in the right tectum. $\boldsymbol{B}$, The effects of tectum ablation and ambient lighting on prey capture performance. Larvae tested in the dark (GFP + Dark) captured significantly fewer paramecia than untreated controls (GFP+ Light). Bilateral tectum ablation (Bilat Tect Abl) impaired performance to a similar degree as testing in darkness, whereas unilateral tectum ablation (Unilat Tect Abl) had an effect intermediate between bilateral ablation and control conditions. There was no significant difference between right- and left-tectum-ablated groups, shown for the $5 \mathrm{~h}$ sampling point only (R.Tec and L.Tec, respectively). C, Prey capture performance of blind lakritz mutants (lak) and wild-type siblings tested in normal lighting or in darkness. The poor performance of blind mutants and larvae tested in darkness relative to controls supports the vision dependence of prey capture.

with Texas Red dextran (see Materials and Methods) and imaged. We found that two identified neurons, MeLr and MeLc, which form a bilaterally symmetric pair situated in the lateral part of the nMLF, extended elaborate dendrites into the ipsilateral tectum
(Fig. 3). RGC axons terminate in four superficial layers of the zebrafish tectum (Xiao et al., 2005). The dendrites of MeLr and $\mathrm{MeLc}$, in contrast, innervated a deeper layer and are concentrated in the medial and caudal boundary of the tectum. This position roughly corresponds to the expected position of neurons in the output layers of the tectum (Meek and Schellart, 1978; Nguyen et al., 1999; Xiao et al., 2005). Based on their morphology, MeLr and $\mathrm{MeLc}$ are thus prime candidates for involvement in tectumdependent visuomotor behaviors.

MeLr and MeLc were laser-ablated under visual control after retrograde labeling from the spinal cord (Fig. 4A,B). Similar procedures have been shown to produce selective killing of targeted neurons (Liu and Fetcho, 1999; O’Malley et al., 2003). After bilateral lesion of MeLc and MeLr, prey capture was substantially decreased relative to injected but unablated controls (mean difference in paramecia decline was $19.16 \% ; p=0.05$ ) (Fig. 4C). The effect of ablating an equal number of non-nMLF RS neurons was also tested to control for laser exposure and to determine whether RS control of prey capture was mediated by a large, distributed network or a smaller, more dedicated group. Control ablations targeted a random subset of four well labeled RS neurons among a group of 14 identified neurons within rhombomeres $4-6$. This group includes the Mauthner neuron. The control ablated group performed similarly to unablated controls in the prey capture assay and significantly better than the bilateral MeLr and MeLc ablated group (mean difference in paramecia decline, $21.91 \%$; $p<0.05$ ) (Fig. 4D). This experiment provides initial evidence that MeLr and MeLc are required for prey capture.

\section{MeLr and MeLc function in a behavioral pathway downstream of the tectum}

Bilateral tectum and bilateral MeLr and MeLc ablation both impaired prey capture, and our anatomical data suggested that MeLr and MeLc are downstream of the tectum. Alternatively, MeLr and MeLc could have a tectum-independent role in prey capture. To test whether the tectum and MeLr/MeLc were linked in the same neural pathway, we performed dual ablations, taking advantage of the fact that MeLr and MeLc dendrites were located only in the ipsilateral tectum. We ablated the retinotectal neuropil on one side, leaving the tectum on the other side intact, and combined this operation with the ablation of either the contralateral or the ipsilateral MeLr/MeLc. If tectum and MeLr/MeLc are in the same pathway, we expect the contralateral ablation to reduce prey capture to a much greater extent than the ipsilateral ablation. The effect of contralateral ablations may in fact be as great as that after bilateral tectum ablation. If tectum and MeLr/ MeLc, however, independently contribute to prey capture, we would expect both combinations to have about the same effect.

We found that fish with contralateral MeLr/MeLc and tectum ablations were significantly impaired in prey capture performance (mean difference from control, 28\%; $p<0.01$ ), whereas fish with ipsilateral ablations were only mildly impaired (Fig. 4C). The ipsilateral group did not show a significant overall difference from the unablated control group but was numerically intermediate between controls and the contralateral ablation group at every sample point, except for the start point (Fig. $4 C$ ). In addition, bilateral MeLr and MeLc ablation appears to have less of an impact than unilateral MeLr and MeLc combined with the contralateral tectum, although the difference is not statistically significant. One possible explanation for this trend is that the tectum relays relevant visual information to RS neurons other than MeLr and MeLc. In that case, bilateral MeLr and MeLc ablation would leave all additional tectoreticular connections intact, whereas the 


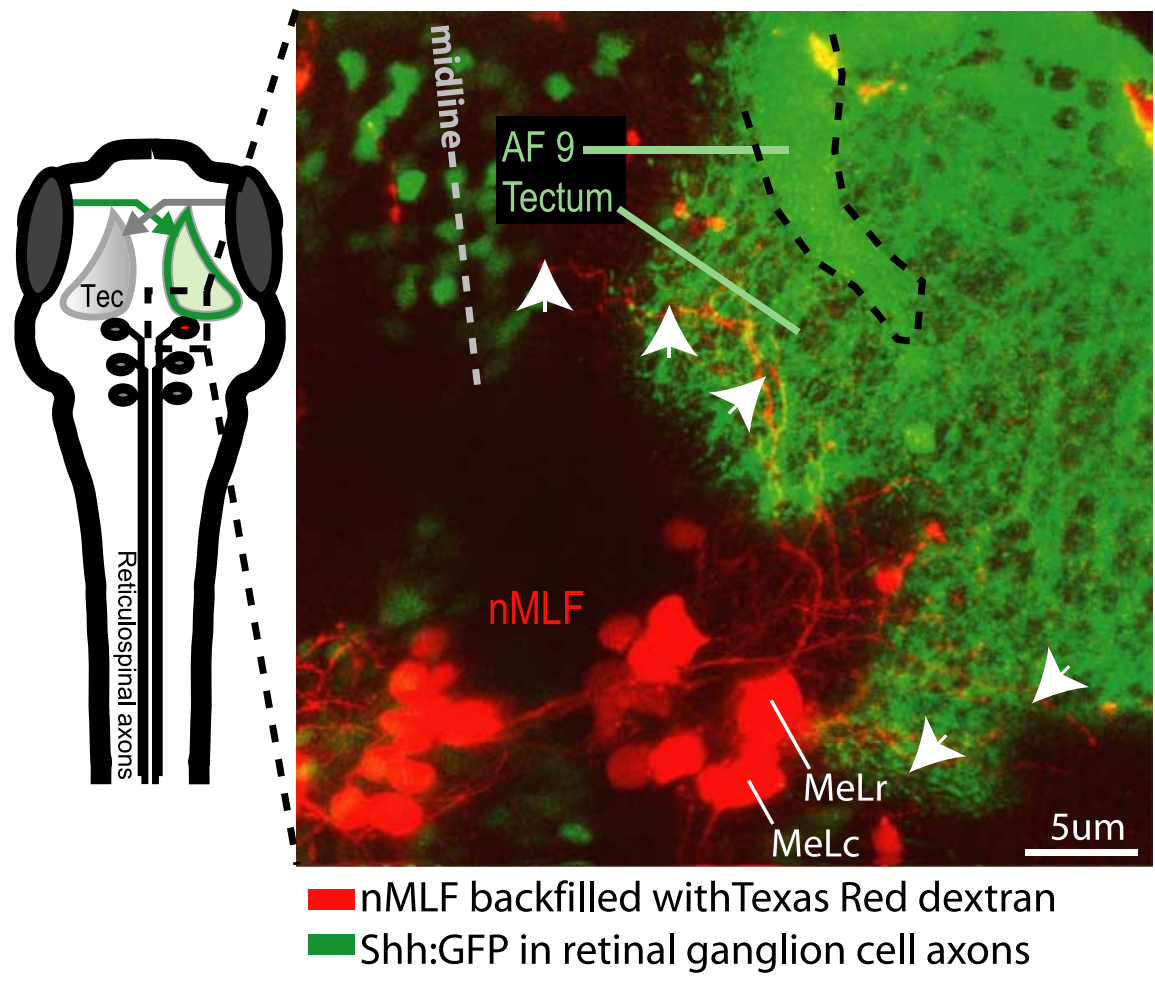

Figure 3. MeL neurons in the nMLF project dendrites into the ventral tectum. A projection of confocal images ( 80 images at 1 $\mu \mathrm{m}$ steps) showing backfilled nMLF neurons (red) and Shh:GFP-expression in RGC axons terminating at the tectum (green). Another retinal ganglion cell arborization field, AF-9, is visible. (AF-9 appears superimposed on the tectum but is in fact ventral to it.) GFP-expressing neurons (green) in the top left of the micrograph belong to the ventral thalamus. MeLc, MeLr, and other nMLF neurons (red) project dendrites into the medioventral region of the deep tectum (arrowheads). RGC axons terminate more superficially and do not overlap with nMLF dendrites, although they appear colocalized in this projection. The diagram to the left indicates the approximate position of the imaged field with respect to the tectum (Tec) and the RS array.

combined contralateral ablation would eliminate all tectoreticular connections on one side of the brain, along with the connection to MeLr and MeL on the other. The combined contralateral ablation would therefore sever more tectoreticular connections than the bilateral MeLc and MeLr ablation. Although MeLr and MeLc may not be the only tectorecipient RS neurons important for prey capture, these experiments firmly place MeLr and MeLc in a tectoreticulospinal pathway for prey capture.

\section{Ablations reduce orientation toward prey}

Prey capture begins with prey recognition and localization, followed by orientation and swimming toward the prey, and culminates in a strike. We asked at what stage the behavior was disrupted by our ablations. To address this question, high-speed (125 frames per second) video recordings of individual prey encounter episodes were analyzed in bilateral tectum-ablated, bilateral MeLr/MeLc-ablated, and control larvae. The first 10 prey encounters, defined as a paramecium moving at close distance $(<5 \mathrm{~mm})$ and within the visual range of the larva, were monitored in each group and analyzed using frame-by-frame playback of video recordings. Swimming responses during prey encounters (10 per experimental group) fell into two specific classes, one termed "orienting swims" and the other termed "routine swims," which closely correspond to the "routine turns" described previously by Budick and O'Malley (2000) (Fig. 5). A distinguishing feature of these two behaviors is the head yaw angle (the angular displacement of the head during successive side-to-side bends of a swim bout): for orienting swims, this angle is much smaller than for routine swims (Fig. 5A-C, three swimming trajectories with the corresponding yaw). Across all three groups, head yaw angle showed a bimodal distribution peaking at $\sim 5^{\circ}$ for orienting swims $(4.80 \pm 0.31$ for successful and $6.08 \pm 0.67$ for unsuccessful captures) and $16^{\circ}$ for routine swims $(15.67 \pm 1.85)$, with a dip in the distribution at $10^{\circ}$ (Fig. 5D). Almost one-half ( 6 of 14) of the orienting swims $\left(<10^{\circ}\right)$ culminated in prey capture, whereas none $(0$ of 16$)$ of the routine swims $\left(>10^{\circ}\right)$ did (Fig. 5D). In addition, orienting swims $\left(<10^{\circ}\right)$ invariably led to an approach (i.e., shortening of the distance between fish and paramecium) at the end of the episode (14 of 14), whereas routine swims $\left(>10^{\circ}\right)$ almost always (14 of 16) resulted in an increase in fish-toparamecium distance, as would be expected from the uncorrelated motion of two objects.

Control larvae oriented in 8 of 10 encounters toward the paramecium and successfully captured the paramecium four of those eight times. Tectum-ablated larvae showed only one orienting swim during the 10 encounters and had no successful capture during a $1 \mathrm{~h}$ observation period. Bilateral MeL-ablated larvae showed five orienting swims, two of which were successful (Fig. 5E). The lack of orienting in the tectum ablation group suggests that these larvae were impaired in their ability to detect paramecia (yaw angle difference, $p<0.001$; two-tailed $t$ test, assuming unequal variance). Orienting responses by MeL-ablated larvae were reduced mildly compared with controls $(p=0.05)$ but not significantly different from the tectum-ablated group $(p=0.75)$. The kinematics of routine and orienting swims were very similar between all three groups with respect to total distance traveled (pairwise comparisons, $p=0.47,0.63$, and 0.69 , respectively) and other parameters. These data show that lesions of tectum and MeL have qualitatively similar effects on the orientation toward prey.

\section{Locomotion and eye movements are unaffected by ablations} of MeLr and MeLc

Could nMLF ablation impair prey capture through an unspecific effect on locomotor control? Zebrafish larvae exhibit characteristic, age-specific patterns of spontaneous swimming activity (Cahill et al., 1998; Drapeau et al., 2002). Therefore, a spontaneous activity assay was used as a measure of general locomotor functioning. Time-lapse videos of spontaneous swimming of groups of larvae were recorded and analyzed in terms of the average percentage of the group that remained in motion (i.e., fish that had changed position from one video frame to the next) across the $20 \mathrm{~min}$ recording period. Blind lakritz mutants showed normal levels of spontaneous swimming frequency and moved similar distances as wild-type sibling controls, suggesting that spontaneous activity level is not vision dependent (percentage of group in motion, $0.84 \pm 0.10$ and $0.94 \pm 0.06$ for lakritz mutants and wild-type controls, respectively). Bilateral tectum-ablated and bilateral MeLr/MeLc-ablated groups also showed normal levels of spontaneous swimming activity relative to their respec- 

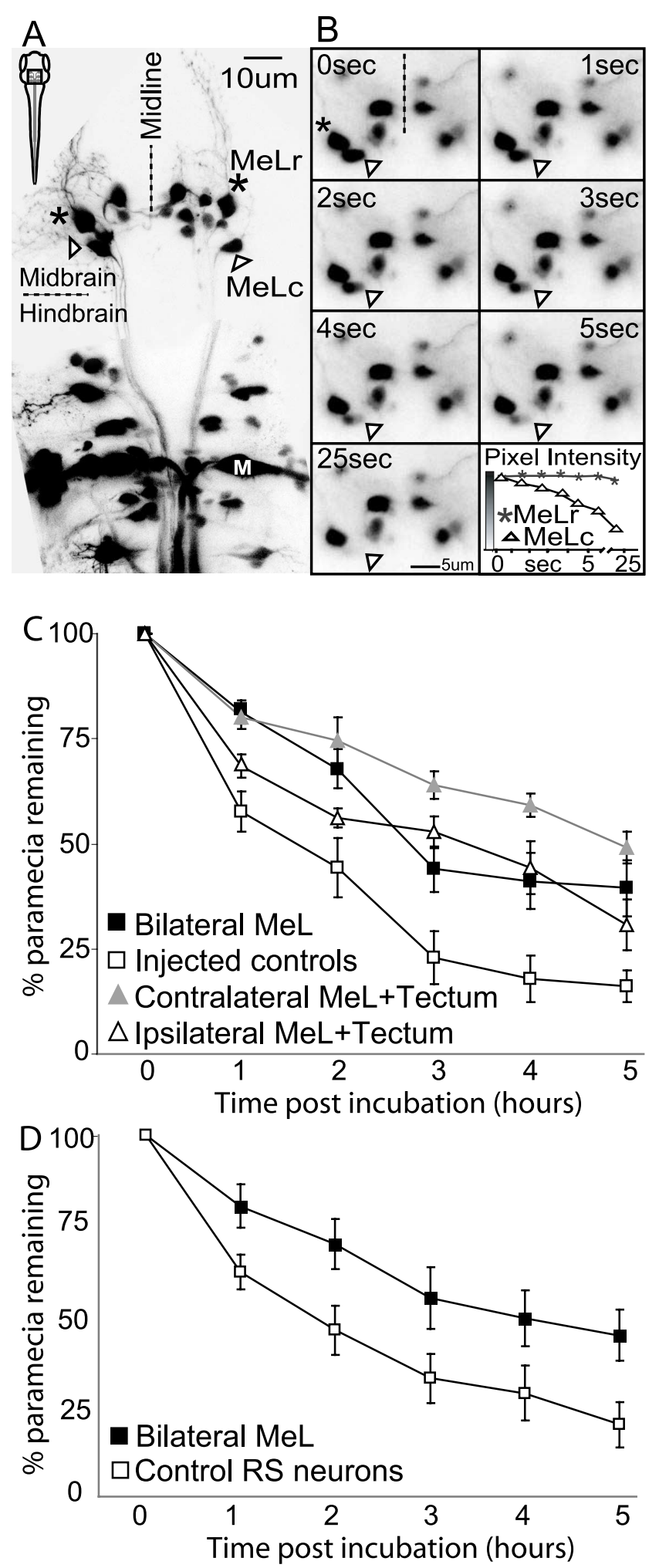

Figure 4. The effect of MeL ablation, and combined MeL and tectum ablation, on prey capture. $\boldsymbol{A}$, Projection of a confocal image stack (35 images at $2 \mu \mathrm{m}$ steps taken through the dorsal surface in a living larva) showing retrograde labeling of RS neurons, including the MeLC and MeLr neurons (arrowhead and asterisk, respectively). The box inside the outline of a larva at the top left indicates the general position of labeled reticulospinal cells. The dendritic projection of the right MeLr neuron is clearly visible, innervating the ventromedial tectum. The $\mathrm{M}$ cell is labeled for reference (M). $\boldsymbol{B}$, A time sequence of nMLF neurons during laser ablation of MeLc. The location of the targeted MeLc neuron and the neighboring MeLr neuron are marked with an arrowhead and asterisk, respectively, as in $\boldsymbol{A}$. Frames $1-6$ are taken at $1 \mathrm{~s}$ intervals, and the seventh frame is taken at $25 \mathrm{~s}$ after the onset of lasing. The plot shows the fluorescence intensity tive control groups (percentage of group in motion, $0.62 \pm 0.10$ for tectum-ablation group, $0.56 \pm 0.06$ for tectum-ablation controls, $0.72 \pm 0.12$ for MeLc/MeLr-ablation group, and $0.64 \pm$ 0.13 for MeLr/MeLc-ablation controls). These lesions also did not impair the OMR, a vision-driven swimming behavior (data not shown). We therefore conclude that spontaneous and visiondriven swimming are not affected by ablation of either the tectum or the lateral nMLF.

In other vertebrates, the control of eye movements has been ascribed to the nMLF, although probably not to those parts of the nMLF that project to the spinal cord. To be certain that our lesion did not affect oculomotor control, we tested the OKR. After unilateral MeLr and MeLc ablation, the OKR was measured using monocular stimulation and comparing the ablated side with the nonablated side within fish. Figure 6 (top) shows example traces of the OKR during stimulation of either eye. The amplitude and frequency of saccades appeared similar for the ablated and intact sides. There was also no overall group difference in the saccade rate or latency to the first saccade between the ablated and intact sides (Fig. 6). These experiments demonstrate that MeLr and MeLc have a specific function in prey capture, although they may play additional roles in other tectum-dependent behaviors.

\section{Discussion}

Zebrafish larvae, at the age of only 1 week after fertilization and with a body length of $<0.5 \mathrm{~cm}$, readily prey on live paramecia, by either chasing the prey or remaining stationary and sucking the prey into their mouths (Borla et al., 2002). Using a quantitative behavioral assay of prey capture performance, we investigated how zebrafish accomplish this feat. We first showed that that prey capture is predominantly visually mediated. Wild-type fish in the dark and blind mutants (lakritz) catch only one-fifth of the paramecia compared with control fish in the light. High-speed video recordings of individual capture episodes revealed that fish often make rapid orienting turns and pursuit swims toward paramecia that were presumably only detectable by vision. During a chase, they hold their heads still (horizontal yaw angle is only $\sim 5^{\circ}$, as opposed to $16^{\circ}$ during routine swims) and steadily decrease the distance to the prey. To delineate the neural pathway underlying prey detection, we then performed laser ablations of candidate CNS structures and tested the lesioned animals using the new assay. The results of these various lesion studies allowed us to make a number of strong predictions about the neural pathway underlying prey capture.

First, we found that integrity of the tectum is indispensable for visually guided prey capture. Tectum-ablated animals almost never oriented toward paramecia in their field of view. This result

$\leftarrow$

(on a 256 level grayscale, indicated by the gradient along the $y$-axis) of the targeted MeLc neuron, as well as for the neighboring MeLr. Loss of fluorescence signal is specific to the targeted MeLc neuron and, after sufficient laser exposure, permanent. C, Prey capture performance in groups with bilateral MeLc and MeLr ablations (Bilateral MeL) or unilateral ablation of these neurons in combination with unilateral tectum ablation on the same (Ipsilateral MeL + Tectum) or opposite (Contralateral MeL + Tectum) sides. Bilateral nMLF ablation and contralateral nMLF plus tectum ablation significantly impaired prey capture performance relative to controls. The ipsilateral nMLF plus tectum ablation group was not significantly different from controls but was numerically intermediate between controls and the crossed ablation group at every sampling point. D, Prey capture performance in a bilateral MeLc and MeLr ablated group (a separate group from that presented in $($ ) and control ablated group in which an equal number of identified RS neurons from within hindbrain rhombomeres $4-6$ were ablated. The control ablated group captured significantly more paramecia than the bilateral MeLc and MeLr group across the $5 \mathrm{~h}$ assay period and was indistinguishable from the unablated group in $\boldsymbol{C}$. 


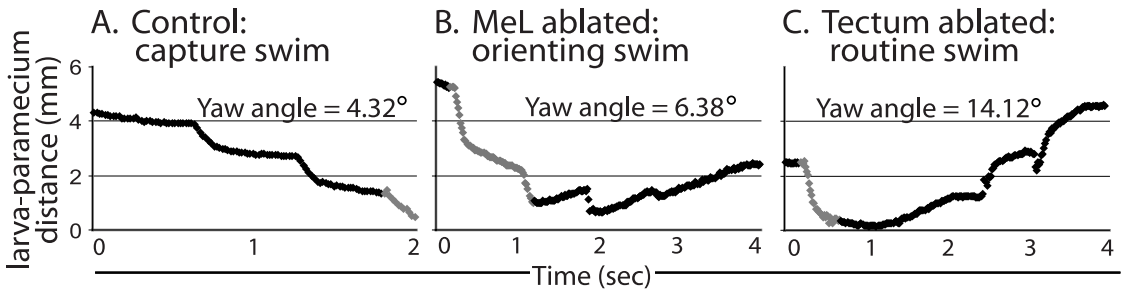

D. Yaw angle distribution

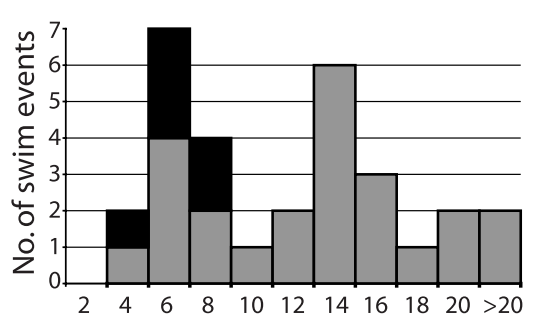

E. Prey encounter behaviors

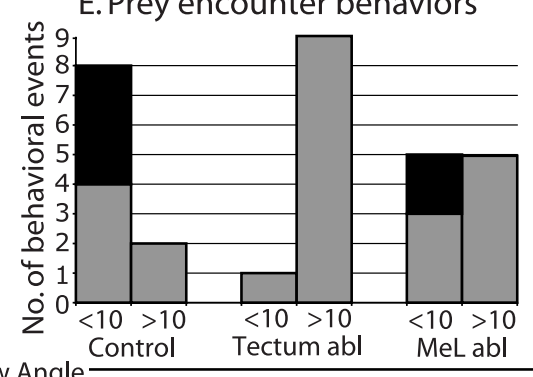

Figure 5. Tectum and MeLr/MeLc ablations disrupt orienting behavior. $\boldsymbol{A}-\boldsymbol{C}$, Three examples of responses to prey. Distances between the larval head and the paramecium on individual prey encounter episodes are plotted ( $x$-axis and $y$-axis labels are the same for each plot). $\boldsymbol{A}$, A successful capture in an unablated control larva. The three periods of rapid decrease in larva-paramecium distance (increases in the slope of the line) reflect three bursts of swimming toward the paramecium, the last of which ends with capture. The yaw value gives the average yaw angle during the final swim burst preceding capture (gray portion of the line). $\boldsymbol{B}$, An orienting swim without prey capture in a bilateral MeLr/MeLc ablated larva. The larva begins with a continuous series of orienting swims toward the paramecia, seen as the rapid decline in larva-paramecia distance in the initial $1 \mathrm{~s}$. The average yaw angle (calculated from the burst of swimming during the gray shaded portion of the line) is low throughout the swim. The slow, steady increase in distance between seconds 2 and 4 reflect the paramecia's movement away from the fish, which had stopped swimming during that period. C, A routine swim (nonresponse) in a tectum-ablated larva. The larva's initial movement brought it very close to the paramecia (probably by chance), but it quickly continued moving away from the paramecia. The yaw angle, calculated from the initial swim burst (gray shaded portion), is much higher than yaw angles typical of orienting swims. $\boldsymbol{D}$, Histogram of yaw angles (in $2^{\circ}$ bins) in all 30 of the prey encounters recorded. The distribution is bimodal, with a peak at $\sim 5^{\circ}$, corresponding to orienting swims, and another peak at $16^{\circ}$, corresponding to routine swims. Almost one-half of the orienting swims resulted in prey capture (black segments). $\boldsymbol{E}$, Distribution of orienting swims $\left(<10^{\circ}\right)$ and routine swims $\left(>10^{\circ}\right)$ during prey encounters, analyzed across ablation groups. In control fish, orienting swims predominate, whereas tectum-ablated (Tectum abl) fish very rarely orient. MeL-ablated (MeL abl) fish are intermediate.

confirms previous electrophysiological and lesioning studies in other vertebrates (Ewert et al., 2001; Lomber et al., 2001; Doubell et al., 2003). For instance, in trout, activation of the tectum elicits orienting movements, the direction and amplitude of which depend on the site of stimulation (Herrero et al., 1998b). However, our study also showed that the tectum is not always necessary for prey consumption, because paramecia numbers still drop at measurable rates in the presence of tectum-ablated (or blind) fish (Fig. 7). This suggests that other sensory modalities, such as olfaction, may also contribute to prey detection.

The fine-grained retinotopic organization and large size of the tectum predispose it for a role in the localization of, and orientation toward, small objects in visual space, a requirement for efficient prey capture. In a previous study, our group showed that OKR, OMR, and visual background adaptation (VBA), a neuroendocrine response to changes in ambient light that acts on skin melanophores through the hypothalamo-pituitary axis, do not depend on an intact tectum (Roeser and Baier, 2003). These experiments, together with the present study, begin to reveal the segregation of processing streams within the zebrafish larval visual system. The tectum is required for prey detection but is dispensable for tasks, such as OKR, OMR, and VBA, that do not require a precise map of visual space.

The central role for the tectum in the vision-driven prey capture observed here does not exclude a function for other retinorecipient areas in prey capture. For instance, in amphibians, pre- tectal pathways are necessary for encoding prey identity (Ewert et al., 2001). Pretectum-lesioned toads snap at all objects indiscriminately. We have not attempted to test the function of any of the four known pretectal areas in zebrafish (Burrill and Easter, 1994), because our prey capture assay, in its current form, does not allow measurement of prey discrimination and is therefore unlikely to detect a behavioral defect after pretectum lesions.

The most striking finding of the current study is the dramatic effect on prey capture seen after ablation of MeLr and MeLc. These four neurons (one MeLr and one MeLc on each side) reside in the lateral portion of the nMLF, the only midbrain structure with descending projections into the spinal cord. MeLr and MeLc extend elaborate dendrites into the deeper layers of the tectum. Here, the cell bodies of tectofugal projection neurons are situated (Meek and Schellart, 1978; Nguyen et al., 1999), which, in other teleosts, have been reported to send a predominantly ipsilateral and excitatory projection to the nMLF (Bosch and Paul, 1993; Herrero et al., 1998a; Niida et al., 1998; Zompa and Dubuc 1998a,b; Isa and Sasaki, 2002; Torres et al., 2002; Perez-Perez et al., 2003). MeLr and MeLc extend axons into the spinal cord, arborizing predominantly in its rostral segments, in which their axon terminals colocalize with motor neurons and interneurons (Gahtan and O'Malley, 2003). Axon branching decreases caudally, but stem axons still reach the most caudal spinal segments. Their morphology thus made MeLr and MeLc excellent candidates for relaying information from the tectum to spinal motor circuits. Bilateral ablation of MeLr and MeLc resulted in a severe prey capture deficit that almost equaled that of bilateral tectum ablations. MeL-ablated fish showed fewer orienting swims than unablated controls, and, even when they did orient, prey capture was rarely successful. Control ablations of other identified RS neurons did not produce significant prey capture deficits. To our knowledge, this finding is one of very few that links a specific behavior to a small number of identified neurons in a vertebrate.

We verified experimentally that MeLr and MeLc acted downstream of the tectum, taking advantage of their exclusively ipsilateral connection. We reasoned that if tectum and nMLF are in the same pathway, combined "crossed" ablations of the tectum on one side and MeLr/MeLc on the other would reduce prey capture to a degree comparable with bilateral ablations of either structure. Ipsilateral, "uncrossed," ablations, in contrast (of tectum and MeLr/MeLc on the same side), should have the same, intermediate effect as unilateral tectum ablations. If tectum and MeLr/MeLc, however, are acting in different pathways we, expect both combinations to have about the same effect (the magnitude of which would be difficult to predict). We find that crossed ablations severely impaired prey capture, similar to bilateral tectum ablations, whereas uncrossed ablations resulted in an intermediate phenotype, similar to unilateral tectum ablations (Fig. 
Control side $(\mathrm{N}=3) \quad$ Ablated side $(\mathrm{N}=3)$
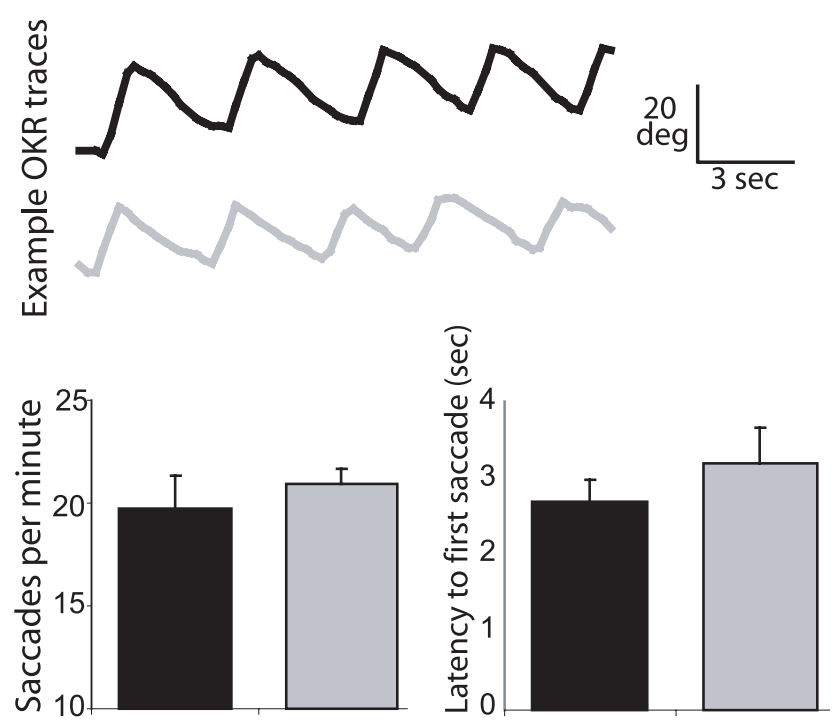

Figure 6. nMLF ablation does not impair the OKR. Comparison of OKR performance for trials in which either the left or right eye was stimulated with a drifting grating stimulus. The three larvae had received unilateral laser ablations of the nMLF, including the MeLr and MeLc neurons, and each eye was subsequently tested on both stimulus directions (temporal-to-nasal and nasal-to-temporal). Therefore, six separate trials were recorded for ablated and intact sides. The top panel shows example OKR traces from a single fish when presented a stimulus to the intact (black trace) or ablated (gray trace) side. The OKR appears normal on both ablated and control trials. The bottom panel shows average saccade frequency (left plot) and response latency before the first saccade (seconds after stimulus onset; right plot) for control and ablated trials, averaging across stimulus direction. Neither comparison of OKR performance showed a significant effect of nMLF ablation. Error bars represent SEM.

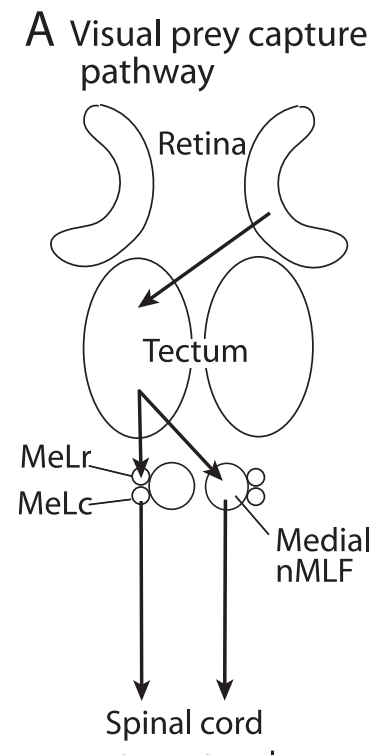

\section{B Effects of ablations on visual prey capture (approx \% of control)}
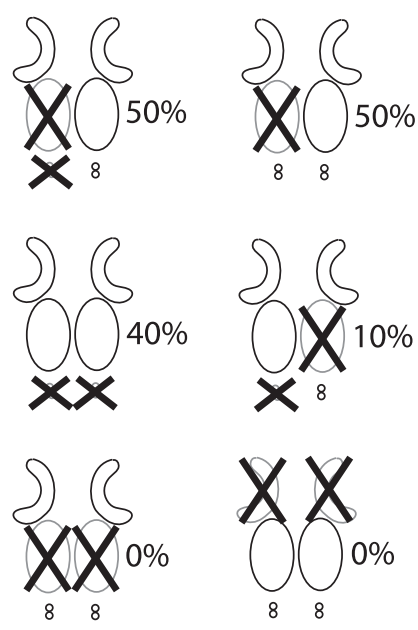

Figure 7. Hypothetical pathway for visual prey capture in zebrafish. $\boldsymbol{A}$, Necessary elements include retina, tectum, nMLF, and spinal cord. $\boldsymbol{B}$, Approximate effects of ablations on prey capture performance.

7B). In other words, ablating the contralateral MeLr/MeLc of fish with just one intact tectum simulated removal of the other tectum, whereas ipsilateral MeLr/MeL ablation did not detectably worsen prey capture performance. This finding strongly suggests that MeLr and MeLc relay signals from the ipsilateral tectum to spinal motor neurons.

Although prey consumption was substantially diminished in MeLr/MeLc-ablated larvae, the severity of this deficit was not as strong as that of bilaterally tectum-ablated larvae. This difference can be explained by additional connections from the tectum to the RS array, including the crossing dendritic projection of medial nMLF cells that innervates the contralateral tectum in zebrafish (Fig. 4A) (Gahtan and O'Malley, 2003) and a descending projection from the tectum to hindbrain RS neurons. We predict that these connections may also participate in the transmission of prey information. Ablation of the entire nMLF, although technically possible (it consists of $\sim 20$ cells on each side of the brain), would be difficult to verify, because not all RS cells are labeled by retrograde tracing in any given animal. Therefore, some cells may escape the operation, and their continued presence after laser treatment would remain undetected.

To show behavioral specificity of MeLr and MeLc, we tested spontaneous swimming, as well as OMR and OKR. All three behaviors were unaffected in ablated larvae. We paid particular attention to the OKR, because studies in fish (Torres et al., 1995), frogs (Kostyk and Grobstein, 1987), and mammals (ButtnerEnnever and Buttner, 1988; Blanks, 1990) have shown that the nMLF influences eye movements. Neurons within the rostral interstitial nMLF provide direct premotor input to the trochlear nerve, which innervates the superior oblique muscle, and other nMLF neurons influence eye movements through a cerebellar circuit (Blanks, 1990). However, our study suggests that the nMLF neurons controlling eye movements (if present in zebrafish) are not identical to MeLr or MeLc.

The nMLF resides in the tegmentum. In amphibians, a tegmental area called the large-celled column (LCC) has been implicated previously in prey recognition (Ewert, 1987; Ewert et al., 2001). It is likely that lesions of the LCC in previous studies encompassed the amphibian homologs of MeLr and MeLc and thus led to similar effects on prey capture to those seen here in fish larvae. What could the function of MeLr and MeLc be? From our high-speed video analysis of prey encounters, it appears that MeL-ablated fish are less able to orient toward prey, mimicking the behavioral deficit after tectum ablation. Thus, MeLc and MeLr may be part of a sparse ensemble of neurons that transmits tectal information on prey location to the motor periphery. Additional studies will be aimed at identifying the other elements of this visuomotor pathway. The fact that the RS neurons in larval zebrafish are individually identifiable, together with our ability to target single neurons for laser ablation, has allowed us to assign a behavioral function to only two identified neurons, rather than hundreds of indistinct cells. Our studies are therefore beginning to trace a dedicated neural pathway, at single-cell resolution, for a complex behavioral program in the vertebrate brain.

\section{References}

Blanks RH (1990) Afferents to the cerebellar flocculus in cat with special reference to pathways conveying vestibular, visual (optokinetic) and oculomotor signals. J Neurocytol 19:628-642.

Borla MA, Palecek B, Budick S, O’Malley DM (2002) Prey capture by larval zebrafish: evidence for fine axial motor control. Brain Behav Evol 60:207-229.

Bosch TJ, Paul DH (1993) Differential responses of single reticulospinal cells to spatially localized stimulation of the optic tectum in a teleost fish, Salmo trutta. Eur J Neurosci 5:742-750.

Budick SA, O'Malley DM (2000) Locomotor repertoire of the larval zebrafish: swimming, turning and prey capture. J Exp Biol 203:2565-2579. Burrill JD, Easter Jr SS (1994) Development of the retinofugal projections in 
the embryonic and larval zebrafish (Brachydanio rerio). J Comp Neurol 346:583-600.

Buttner-Ennever JA, Buttner U (1988) Neuroanatomy of the oculomotor system. The reticular formation. Rev Oculomot Res 2:119-176.

Cahill GM, Hurd MW, Batchelor MM (1998) Circadian rhythmicity in the locomotor activity of larval zebrafish. NeuroReport 9:3445-3449.

Doubell TP, Skaliora I, Baron J, King AJ (2003) Functional connectivity between the superficial and deeper layers of the superior colliculus: an anatomical substrate for sensorimotor integration. J Neurosci 23:6596-6607.

Drapeau P, Saint-Amant L, Buss RR, Chong M, McDearmid JR, Brustein E (2002) Development of the locomotor network in zebrafish. Prog Neurobiol 68:85-111.

Eaton RC, DiDomenico R, Nissanov J (1991) Role of the Mauthner cell in sensorimotor integration by the brain stem escape network. Brain Behav Evol 37:272-285.

Eaton RC, Lee RK, Foreman MB (2001) The Mauthner cell and other identified neurons of the brainstem escape network of fish. Prog Neurobiol 63:467-485.

Edwards DH, Heitler WJ, Krasne FB (1999) Fifty years of a command neuron: the neurobiology of escape behavior in the crayfish. Trends Neurosci 22:153-161.

Ewert JP (1987) Neuroethology of releasing mechanisms: prey catching in toads. Behav Brain Sci 10:337-405.

Ewert JP, Buxbaum-Conradi H, Dreisvogt F, Glagow M, Merkel-Harff C, Rottgen A, Schurg-Pfeiffer E, Schwippert WW (2001) Neural modulation of visuomotor functions underlying prey-catching behaviour in anurans: perception, attention, motor performance, learning. Comp Biochem Physiol A Mol Integr Physiol 28:417-461.

Faber DS, Fetcho JR, Korn H (1989) Neuronal networks underlying the escape response in goldfish. General implications for motor control. Ann NY Acad Sci 563:11-33.

Gahtan E, Baier H (2004) Of lasers, mutants, and see-through brains: functional neuroanatomy in zebrafish. J Neurobiol 59:147-161.

Gahtan E, O'Malley DM (2003) Visually guided injection of identified reticulospinal neurons in zebrafish: a survey of spinal arborization patterns. J Comp Neurol 459:186-200.

Gahtan E, Sankrithi N, Campos JB, O’Malley DM (2002) Evidence for a widespread brain stem escape network in larval zebrafish. J Neurophysiol $87: 608-614$.

Herrero L, Corvisier J, Hardy O, Torres B (1998a) Influence of the tectal zone on the distribution of synaptic boutons in the brainstem of goldfish. J Comp Neurol 401:411-428.

Herrero L, Rodriguez F, Salas C, Torres B (1998b) Tail and eye movements evoked by electrical microstimulation of the optic tectum in goldfish. Exp Brain Res 120:291-305.

Isa T, Sasaki S (2002) Brainstem control of head movements during orienting; organization of the premotor circuits. Prog Neurobiol 66:205-241.

Kay JN, Finger-Baier KC, Roeser T, Staub W, Baier H (2001) Retinal ganglion cell genesis requires lakritz, a zebrafish atonal homolog. Neuron 30:725-736.

Kostyk SK, Grobstein P (1987) Neuronal organization underlying visually elicited prey orienting in the frog. II. Anatomical studies on the laterality of central projections. Neuroscience 21:57-82.
Kupfermann I, Weiss KR (2001) Motor program selection in simple model systems. Curr Opin Neurobiol 11:673-677.

Liu KS, Fetcho JR (1999) Laser ablations reveal functional relationships of segmental hindbrain neurons in zebrafish. Neuron 23:325-335.

Lomber SG, Payne BR, Cornwell P (2001) Role of the superior colliculus in analyses of space: superficial and intermediate layer contributions to visual orienting, auditory orienting, and visuospatial discriminations during unilateral and bilateral deactivations. J Comp Neurol 441:44-57.

Meek J, Schellart NA (1978) A Golgi study of goldfish optic tectum. J Comp Neurol 182:89-122.

Metzner W, Juranek J (1997) A sensory brain map for each behavior? Proc Natl Acad Sci USA 94:14798-14803.

Neumann CJ, Nuesslein-Volhard C (2000) Patterning of the zebrafish retina by a wave of sonic hedgehog activity. Science 289:2137-2139.

Nguyen V, Deschet K, Henrich T, Godet E, Joly JS, Wittbrodt J, Chourrout D, Bourrat F (1999) Morphogenesis of the optic tectum in the medaka (Oryzias latipes): a morphological and molecular study, with special emphasis on cell proliferation. J Comp Neurol 413:385-404.

Niida A, Ohono T, Iwata KS (1998) Efferent tectal cells of crucian carp: physiology and morphology. Brain Res Bull 22:389-398.

O’Malley DM, Kao YH, Fetcho JR (1996) Imaging the functional organization of zebrafish hindbrain segments during escape behaviors. Neuron 17:1145-1155.

O’Malley DM, Zhou Q, Gahtan E (2003) Probing neural circuits in the zebrafish: a suite of optical techniques. Methods [Erratum (2003) 30:353355] 30:49-63.

Orger MB, Gahtan E, Muto A, Page-McCaw P, Smear MC, Baier H (2004) Behavioral screening assays in zebrafish. Methods Cell Biol 77:55-68.

Perez-Perez MP, Luque MA, Herrero L, Nunez-Abades PA, Torres B (2003) Connectivity of the goldfish optic tectum with the mesencephalic and rhombencephalic reticular formation. Exp Brain Res 151:123-135.

Roeser T, Baier H (2003) Visuomotor behaviors in larval zebrafish after GFP-guided laser ablation of the optic tectum. J Neurosci 23:3726-3734.

Sewards TV, Sewards MA (2002) Innate visual object recognition in vertebrates: some proposed pathways and mechanisms. Comp Biochem Physiol A Mol Integr Physiol 132:861-891.

Svoboda KR, Fetcho JR (1996) Interactions between the neural networks for escape and swimming in goldfish. J Neurosci 16:843-852.

Torres B, Fernandez S, Rodriguez F, Salas C (1995) Distribution of neurons projecting to the trochlear nucleus in goldfish (Carassius auratus). Brain Behav Evol 45:272-285.

Torres B, Perez-Perez MP, Herrero L, Ligero M, Nunez-Abades PA (2002) Neural substrata underlying tectal eye movement codification in goldfish. Brain Res Bull 57:345-348.

Xiao T, Roeser T, Staub W, Baier H (2005) A GFP-based genetic screen reveals mutations that disrupt the architecture of the zebrafish retinotectal projection. Development 132:2955-2967.

Zompa IC, Dubuc R (1998a) Diencephalic and mesencephalic projections to rhombencephalic reticular nuclei in lampreys. Brain Res 802:27-54.

Zompa IC, Dubuc R (1998b) Electrophysiological and neuropharmacological study of tectoreticular pathways in lampreys. Brain Res 804:238-252.

Zottoli SJ, Hordes AR, Faber DS (1987) Localization of optic tectal input to the ventral dendrite of the goldfish Mauthner cell. Brain Res 401:113-121. 